\title{
Relationship between coercivity and magnetic moment of superparamagnetic particles with dipolar interaction
}

\author{
V. Franco, C. F. Conde, and A. Conde \\ Dpto. Física de la Materia Condensada, ICMSE-CSIC, Universidad de Sevilla, P. O. Box 1065, 41080 Sevilla, Spain \\ L. F. Kiss \\ Research Institute for Solid State Physics and Optics, Hungarian Academy of Sciences, P. O. Box 49, 1525 Budapest, Hungary
}

(Received 28 July 2005; revised manuscript received 8 September 2005; published 22 November 2005)

\begin{abstract}
The temperature dependence of the hysteresis loops of Nanoperm-type alloys has been studied. In the high-temperature region above the coercivity maximum, the response of the system can be modeled as that of dipolar-interaction superparamagnetic particles, considering a mean interaction field. Special attention has been paid to the influence of the particle size distribution on the applicability of the mean-field model. The two main effects of the dipolar interaction (coercivity and distortion of the thermal dependence of the apparent magnetic moment) have been correlated.
\end{abstract}

DOI: $10.1103 /$ PhysRevB.72.174424

PACS number(s): 75.50.Tt, 75.50.Kj, 75.10.Nr

\section{INTRODUCTION}

The magnetic characterization of fine particles is a field of current interest. Not only do the properties of these particles deviate from those of their bulk counterparts, which is an interesting subject of study in fundamental physics, but also these particles are promising for numerous technological applications, ranging from data storage to biotechnology. ${ }^{1}$

As the characteristic size of the material decreases, interparticle interactions (exchange and/or dipolar) get increasing importance, originating numerous scientific studies. ${ }^{2,3}$ Depending on the type of material, its microstructure, and the operating temperature, the type of interparticle interaction that controls its magnetic behavior can be different. For example, centering our attention on magnetic recording media, while in the case of thin films the dominant interaction is exchange coupling, for particulate media the leading role is played by the magnetostatic interaction. ${ }^{4}$ A special type of materials are the nanocrystalline alloys of the Finemet and Nanoperm families. In these cases, ferromagnetic nanocrystalline particles are embedded in a residual amorphous matrix with a relatively low Curie temperature $\left(\sim 300^{\circ} \mathrm{C}\right)$. Provided that the crystalline fraction is small enough, the relevant interaction between the nanocrystals can be controlled by simply changing the measuring temperature, making these systems particularly interesting for analyzing the influence of the different interaction types on the magnetic characteristics of samples. The main virtue of these systems is that their microstructure remains unchanged for the different interaction regimes.

Recently, the magnetic hysteresis observed in granular magnetic materials at temperatures above the superparamagnetic blocking temperature of the particles has been modeled by introducing a memory term inside the argument of the Langevin function, related to a mean dipolar interaction field. ${ }^{5}$ This model has been successfully applied to different kind of granular materials, such as $\mathrm{Co}-\mathrm{Cu}$ alloys ${ }^{5} \mathrm{Co}$ nanoparticles embedded in a $\mathrm{SiO}_{2}$ matrix, ${ }^{6}$ and Finemet-type alloys. ${ }^{7}$
A remarkable experimental fact is that for superparamagnetic particles with dipolar interaction, the magnetic moment calculated from the Langevin fitting of the anhysteretic magnetization curve does not correspond to the real magnetic moment of the particles. This discrepancy is evidenced by analyzing the thermal dependence of the calculated magnetic moment of a system of thermally stable particles, a moment with an apparent increase with temperature. This apparently anomalous effect of the dipolar interaction between the particles has been modeled by using an effective temperature related to the rms dipolar energy. ${ }^{8}$ The application of this model to Finemet-type alloys provided a first approach to relate both effects of the dipolar interaction between the particles. $^{7}$

It is important to mention that geometrical factors can play a relevant role in the effects of the dipolar interaction between particles. ${ }^{9}$ Specifically, the existence of a grain size distribution in the studied sample can affect the applicability of the mean-field model. In the case of $\mathrm{Co}-\mathrm{Cu}$ alloys, grain size distributions were taken into account by considering that the response of the system to the additional excitation field was that of an average moment. ${ }^{5}$ In order to check the applicability of that assumption to other systems, we have studied Cr- and Cr-Mo-substituted Nanoperm-type alloys at the early stages of nanocrystallization. The presence of Mo in the alloy composition reduces the Curie temperature of the amorphous alloy close to room temperature, facilitating the exchange decoupling between the nanocrystals, ${ }^{10}$ while $\mathrm{Cr}$ has a similar effect in the Finemet-type alloys. ${ }^{7}$ It will be shown that there are cases where the response of a system with a distribution of particle sizes cannot be represented by the response of an average system, although the mean dipolar interaction field retains its meaning and usefulness.

The structure of this paper will be the following. Section II summarizes the experimental techniques used. Section III presents the experimental results regarding the devitrification process of the studied alloys and the thermal dependence of the hysteresis loops, which will be subsequently modeled in Sec. IV. Initially, an attempt is made to model the loops with 


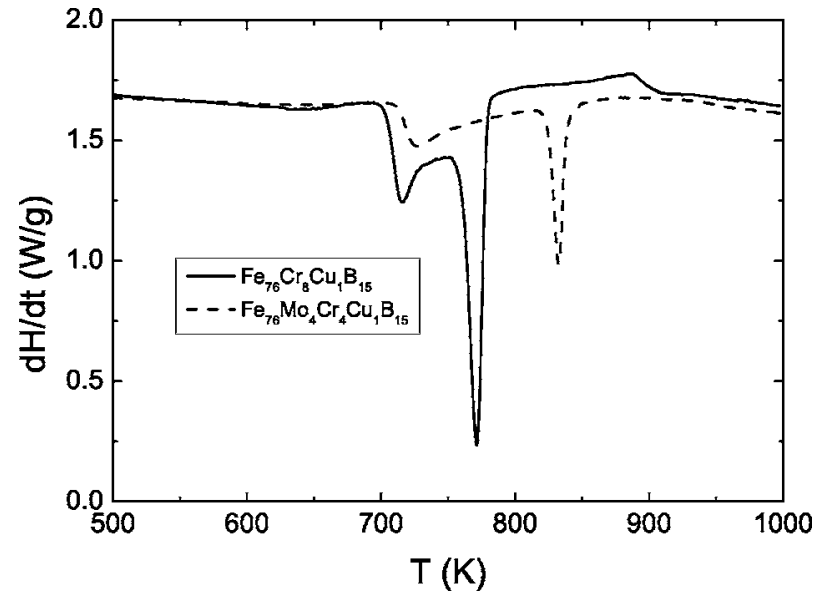

FIG. 1. Differential scanning calorimetry curves, taken at $10 \mathrm{~K} / \mathrm{min}$, of the as-cast $\mathrm{Fe}_{76} \mathrm{Cr}_{8} \mathrm{Cu}_{1} \mathrm{~B}_{15}$ and $\mathrm{Fe}_{76} \mathrm{Cr}_{4} \mathrm{Mo}_{4} \mathrm{Cu}_{1} \mathrm{~B}_{15}$ samples.

the use of a single average magnetic moment. After realizing that in one case this approach (i.e., using an average memory function) fails, the way in which particle size distributions are taken into account in the hysteresis model is modified, resulting in a much better fit to the loops. Section V makes the relation between both effects of the dipolar interaction more precise. Finally, conclusions are drawn.

\section{EXPERIMENT}

Amorphous ribbons of the nominal composition $\mathrm{Fe}_{76} \mathrm{Cr}_{8} \mathrm{Cu}_{1} \mathrm{~B}_{15}$ and $\mathrm{Fe}_{76} \mathrm{Cr}_{4} \mathrm{Mo}_{4} \mathrm{Cu}_{1} \mathrm{~B}_{15}$ were prepared by single roller melt spinning. Their amorphous character was checked by $\mathrm{x}$-ray diffraction. The devitrification process was studied with a Perking-Elmer DSC-7 differential scanning calorimeter (DSC). The evolution of the Curie temperature of the nanostructured material during devitrification was determined with the help of a Perkin-Elmer TGA-7 thermobalance applying the field of a small permanent magnet. To obtain the desired microstructure, samples were heated at $10 \mathrm{~K} / \mathrm{min}$ up to the target temperature. The thermal dependence of the hysteresis loops was measured by a superconducting quantum interference device magnetometer (Quantum Design MPMS-5S) from 300 up to $700 \mathrm{~K}$. Transmission electron microscopy TEM observations were performed on a Philips CM-200 electron microscope with an accelerating voltage of $200 \mathrm{kV}$.

\section{RESULTS}

\section{A. Devitrification process}

The devitrification process of the studied alloys is similar to that observed in nanocrystalline soft magnetic materials of the Nanoperm family. ${ }^{10-13}$ It takes place in two main stages, as evidenced in the differential scanning calorimetry plot (Fig. 1). The first one corresponds to the appearance of a bcc Fe nanophase embedded in the residual amorphous matrix, while the second one, after which the sample is fully crystallized, is associated with the formation of boride-type

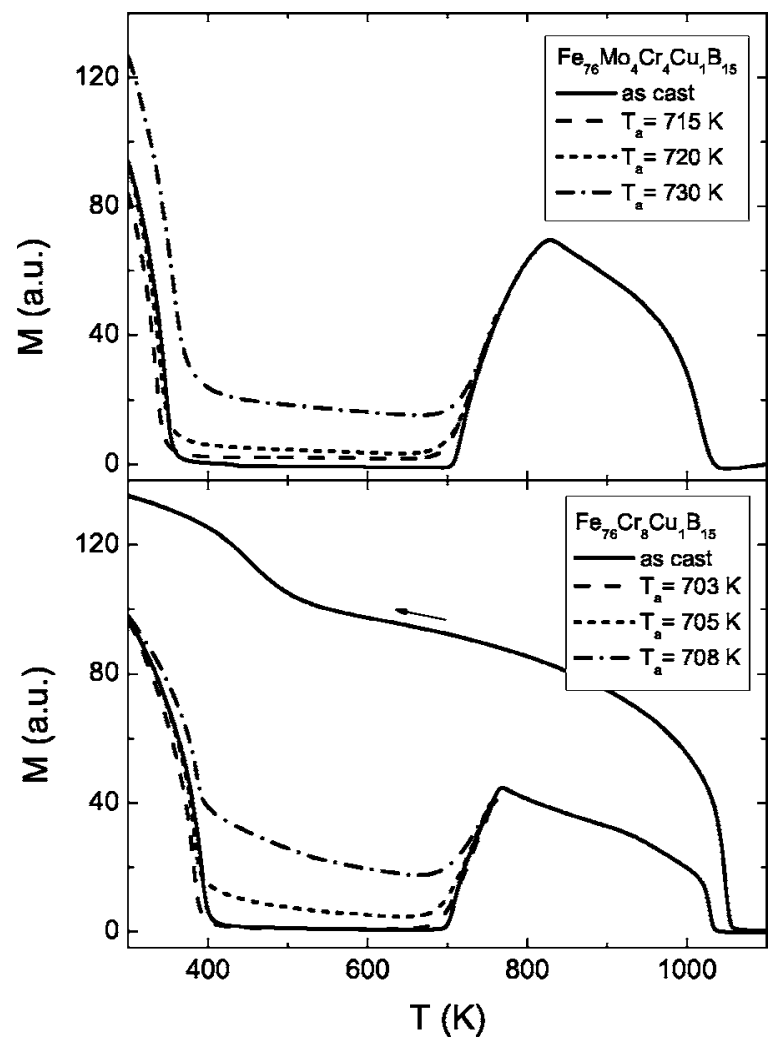

FIG. 2. Temperature dependence of the low-field magnetization of the as-cast and preheated samples (up to the indicated temperature). For the Mo-free alloy, the cooling process of a fully crystallized sample is also recorded.

phases and includes the recrystallization of some preexisting bcc Fe crystals. ${ }^{14,15}$ The presence of Mo in the alloy produces a stabilization of the amorphous phase against nanocrystallization, as evidenced by the displacement to higher temperatures of the nanocrystallization onset, as well as the stabilization of the nanophase by increasing the onset temperature of the second crystallization stage. This latter effect prevents the overlapping of the two crystallization stages for the Mo-containing alloy.

The temperature evolution of the low-field magnetization of the as-cast alloys shows (Fig. 2) a vanishing magnetization $(M)$ at the Curie temperature $\left(T_{C}\right)$ of the amorphous phase, an increase in $M$ associated with the onset of nanocrystallization, and a further decrease corresponding to the recrystallization process and then to the $T_{C}$ of the bcc $\mathrm{Fe}$ phase. In the case of the Mo-free alloy, the thermomagnetic curve recorded during the cooling after full crystallization is also plotted, showing the Curie temperature of the final magnetic crystalline phases: bcc Fe and boride phases. For both alloys, the thermomagnetic scans of the preheated samples indicate that annealing the samples at moderated temperatures produces a slight reduction in the $T_{C}$ of the amorphous alloy due to structural relaxation. As nanocrystallization begins, $T_{C}$ increases progressively. The main difference between the two alloys is a higher $T_{C}$ of the amorphous phase in the case of the Mo-free alloy. Table I contains the peak temperatures of the DSC exothermal maxima, as well as the evolution of the Curie temperature of the residual amorphous 
TABLE I. Peak temperatures of the DSC curves for the studied alloys, and Curie temperatures of the (residual) amorphous matrix of the samples preheated up to the temperature indicated in brackets, derived from the magnetization curves.

\begin{tabular}{lll}
\hline \hline & $\mathrm{Fe}_{76} \mathrm{Cr}_{8} \mathrm{Cu}_{1} \mathrm{~B}_{15}$ & $\mathrm{Fe}_{76} \mathrm{Cr}_{4} \mathrm{Mo}_{4} \mathrm{Cu}_{1} \mathrm{~B}_{15}$ \\
\hline$T_{p 1}(\mathrm{~K})$ & 715 & 727 \\
$T_{p 2}(\mathrm{~K})$ & 771 & 832 \\
$T_{c}(\mathrm{am}).(\mathrm{K})[$ As cast $]$ & 392 & 346 \\
$T_{c}(\mathrm{am}).(\mathrm{K})[703 \mathrm{~K}]$ & 382 & \\
$T_{c}(\mathrm{am}).(\mathrm{K})[705 \mathrm{~K}]$ & 383 & \\
$T_{c}(\mathrm{am}).(\mathrm{K})[708 \mathrm{~K}]$ & 385 & \\
$T_{c}(\mathrm{am}).(\mathrm{K})[715 \mathrm{~K}]$ & & 335 \\
$T_{c}(\mathrm{am}).(\mathrm{K})[720 \mathrm{~K}]$ & & 341 \\
$T_{c}(\mathrm{am}).(\mathrm{K})[730 \mathrm{~K}]$ & & 358 \\
\hline \hline
\end{tabular}

matrix as a function of the previous thermal treatment.

\section{B. Temperature dependence of hysteresis}

Figure 3 shows the thermal dependence of coercivity of the studied nanocrystalline samples. The temperature range has been selected to avoid the evolution of the microstructure of the samples during the measurement process. Three different regimes can be detected in the curves. At low temperatures, the coercivity has a small value due to the exchange coupling between the nanocrystals through the ferromagnetic amorphous matrix, which produces the averaging out of the magnetocrystalline anisotropy. ${ }^{16}$ As the Curie temperature of the matrix is approached, the coercivity increases as the matrix cannot transmit the exchange so efficiently. ${ }^{17}$ At higher temperatures, after a maximum, the coercivity tends to diminish. This last regime has been explained in soft magnetic nanocrystalline materials as a transition to superparamagnetism, ${ }^{18}$ controlled by the dipolar interaction between the nanocrystals, which causes the progressive decrease of the coercivity. It has been shown that Finemet-type alloys containing Cr (Refs. 7, 18, and 19) or Cr-Mo (Ref. 20)

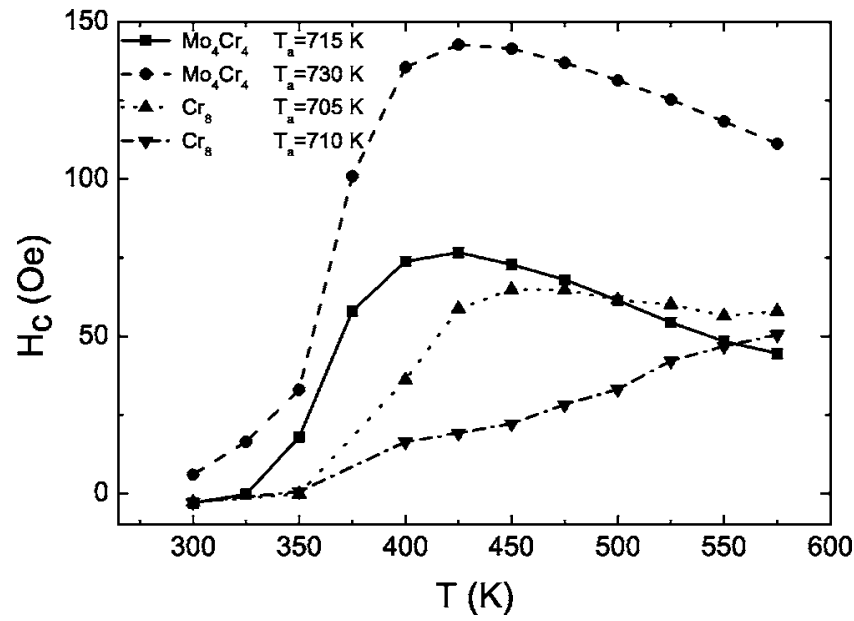

FIG. 3. Temperature dependence of the coercivity of preheated samples of the studied alloys. show fully superparamagnetic behavior at temperatures above the Curie temperature of the remaining amorphous matrix. Also, for typical (FeSiBCuNb) Finemet alloys, superparamagnetism occurs at the early stages of nanocrystallization, ${ }^{21}$ which has been interpreted as a demonstration that superparamagnetism is a general feature in soft magnetic nanocrystalline alloys for low enough crystalline fractions. For Nanoperm alloys, with a similar microstructure to that of Finemet-type alloys (in the sense that $\mathrm{Fe}$ nanocrystals- $-\mathrm{Fe}, \mathrm{Si}$ in the case of Finemet-are embedded in a remaining amorphous matrix), superparamagnetic behavior has been reported. ${ }^{22}$ Therefore, although in the experimental data the complete superparamagnetic regime is not reached (the coercivity does not fall to zero), it is reasonable to consider that the mentioned coercivity decrease after the maximum is evidence of the transition to superparamagnetism. As the nanocrystalline material is metastable, it is not possible to increase further the measuring temperature without altering the microstructure of the sample, preventing us from observing the zero coercivity.

As the crystalline fraction increases, the maximum value of the coercivity increases due to the stronger interaction between the particles. ${ }^{7}$ Also, the temperature at which the maximum is detected shifts to higher temperatures, in correlation with the increase of the Curie temperature of the remaining amorphous matrix mentioned above.

The essential difference between the behavior of the two alloys is that while a maximum in coercivity can be detected for the Mo-containing alloy, the Mo-free samples show either a continuous increase in coercivity, or a plateau. This can also be related to the higher Curie temperature of the amorphous matrix in the latter case. Therefore, the Mo-free samples will not be adequate for analyzing the dipolar interaction between the nanoparticles.

\section{MODELING THE HYSTERESIS LOOPS}

\section{A. Using a single magnetic moment}

In the temperature range above the coercivity maximum, the hysteresis loop of the samples can be analyzed using a mean-field model. ${ }^{5}$ It considers the effect of the dipolar interactions between the superparamagnetic particles as a memory function and can be summarized as follows. The anhysteretic reduced magnetization of the sample, $m$, is defined as the half sum of the two branches of the reduced hysteresis loop. $\Delta$ is defined as the half difference of the two branches of the reduced hysteresis loop. The skeleton of the loop should correspond to a Langevin function (the extension to a distribution of particle sizes will be considered in next section), as this is the behavior of the particles in the absence of interactions. For major hysteresis loops, the only ones considered in this paper, the memory function $\delta(m)$ depends on the anhysteretic reduced magnetization of the sample, $m$. The branches of the major loop can be described as

$$
m_{ \pm} \approx L\left(\frac{\mu H}{k T} \pm \delta(m)\right)
$$

where $\mu$ is the magnetic moment of the particle (which will be referred to later as the "apparent magnetic moment"), $H$ is 
the applied field, $T$ is the measuring temperature, and $k$ is the Boltzmann constant.

The memory function can be rewritten as a function of an effective interaction field $H_{0}$ (related to the root mean square of the dipolar field) and a "cutoff" function $F(m)$ as follows:

$$
\delta(m)=\frac{\mu H_{0}}{k T} F(m) .
$$

The magnetic moment of the particles can be calculated through a simple Langevin fitting to the anhysteretic curve. Meanwhile, the reduced half difference of the two branches $[\Delta(m)]$ is a measure of how the hysteresis loop separates from the superparamagnetic skeleton. Therefore, it should be related to the cutoff function. After some manipulations, defining $m_{r}$ as the reduced remanence of a major loop, the cutoff function can be obtained as

$$
F(m)=\frac{\Delta(m)}{3 m_{r} u^{\prime}(m)},
$$

where $u^{\prime}(m)$ is the first derivative of the Langevin function with respect to its argument. An alternative expression for the cutoff function can also be obtained:

$$
F=3 \sqrt{3} u^{\prime}(m)\left(1-\frac{2 m k T}{\mu H}-m^{2}\right)^{1 / 2} .
$$

In this case, it has to be noted that the $F(m)$ curve can be generated if the magnetic moment of the particles is known.

The different parameters required for reconstructing the branches of a major loop are obtained as follows. The magnetic moment is determined from the Langevin fitting to the anhysteretic curve; the cutoff function can be obtained either from the reduced half difference of the branches through Eq. (3) (experimental $F$ ), or from Eq. (4) (predicted $F$ ) once the magnetic moment is known, and finally, the interaction field is calculated from

$$
m_{r}=\frac{1}{3} \frac{\mu H_{0}}{k T} .
$$

The major loop is obtained as

$$
m_{ \pm}=L\left(\frac{\mu H}{k T} \pm \frac{\mu H_{0}}{k T} F(m)\right) .
$$

As indicated in the previous section, superparamagnetism is a common feature of this kind of soft magnetic nanocrstalline alloy at temperatures above the coercivity maximum. However, the existence of dipolar interactions between the nanoparticles causes a gradual transition to the superparamagnetic regime. Following the described procedure, the hysteresis loops of the Mo-containing sample preheated up to $730 \mathrm{~K}$ can be modeled with remarkable agreement in the temperature range starting from the coercivity maximum. As an example, Fig. 4 shows the fit of the anhysteretic magnetization curve, measured at $475 \mathrm{~K}$, of the Mocontaining alloy preheated up to $730 \mathrm{~K}$. In this case, a Langevin function plus a linear contribution were fitted to the curve. The reason for using this linear part (with a magnetization evolving as $\chi H$ ), whose value is of the order of

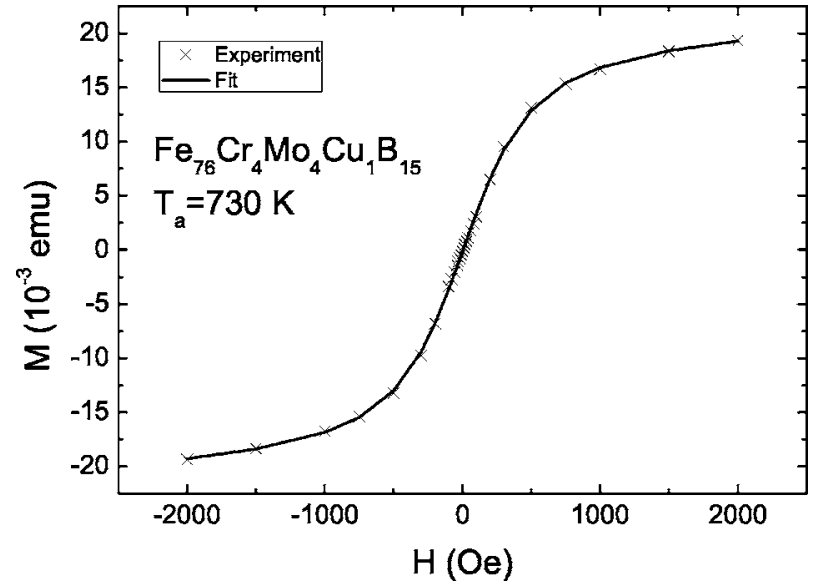

FIG. 4. Anhysteretic magnetization curve obtained from the half sum of the hysteresis loop branches of the $\mathrm{Fe}_{76} \mathrm{Cr}_{4} \mathrm{Mo}_{4} \mathrm{Cu}_{1} \mathrm{~B}_{15}$ alloy preheated up to $730 \mathrm{~K}$ and measured at $475 \mathrm{~K}$. The line corresponds to a Langevin fit.

$10^{-7} \mathrm{emu} / \mathrm{Oe}$, is to take into account the magnetic signal not coming from the nanoparticles but from the paramagnetic matrix and the sampleholder. Figure 5 shows the experimental $\Delta(m)$ data together with those obtained from the predicted $F$, for the samples preheated up to 715 and $730 \mathrm{~K}$. Figure 6 shows the experimental (crosses) and calculated loops of those samples.

Care has to be taken when using Eq. (3) for obtaining the cutoff function (experimental $F$ ). While for the higher annealing temperature there is no difference between the fitted loops using the experimental $F$ and those provided by Eq. (4) (predicted $F$ ), for the sample annealed at the lower temperature the use of the predicted $F$ shows discrepancies between

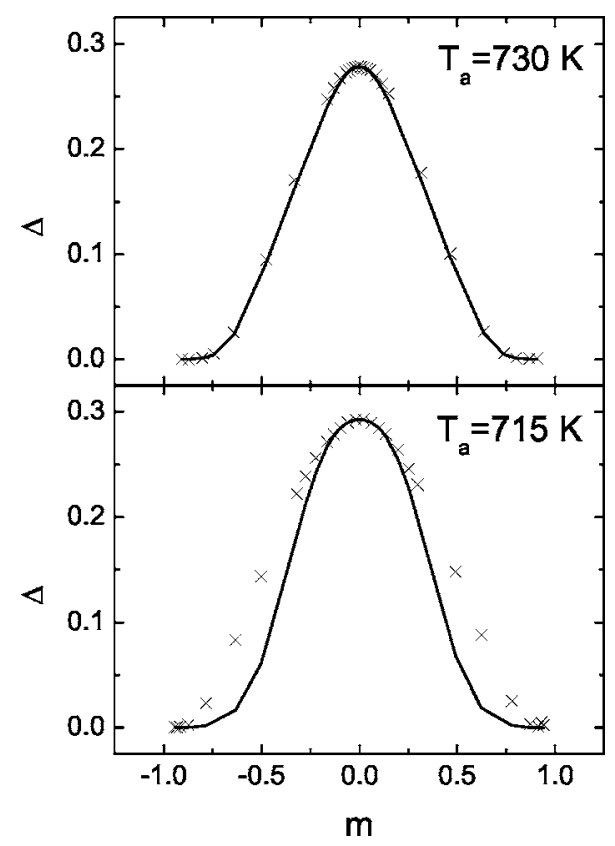

FIG. 5. $\Delta(m)$ of the $\mathrm{Fe}_{76} \mathrm{Cr}_{4} \mathrm{Mo}_{4} \mathrm{Cu}_{1} \mathrm{~B}_{15}$ samples preheated up to 715 and $730 \mathrm{~K}$ measured at $475 \mathrm{~K}$ : experimental data (crosses); calculated from the predicted $F$ [Eq. (4)] (lines). 


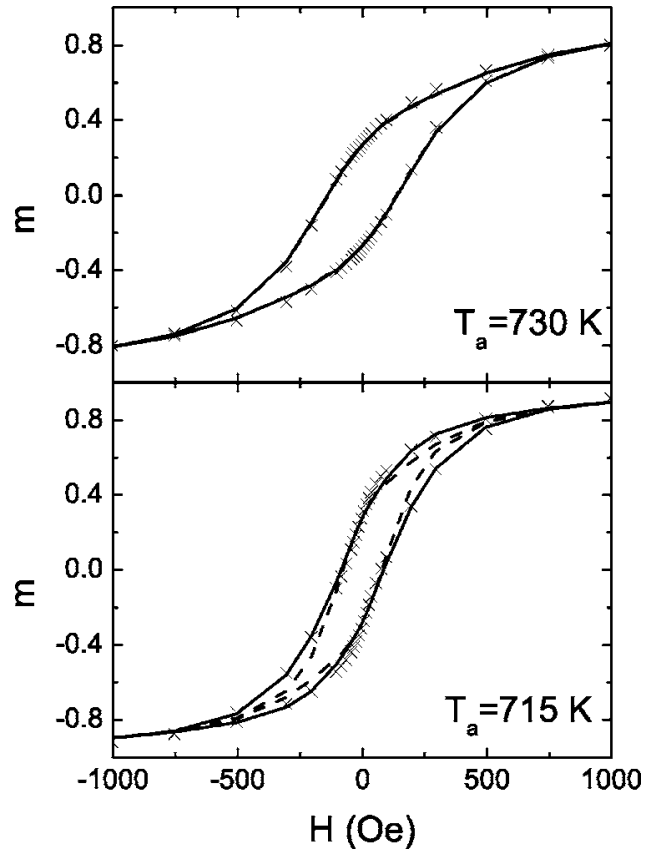

FIG. 6. Reduced hysteresis loops of the $\mathrm{Fe}_{76} \mathrm{Cr}_{4} \mathrm{Mo}_{4} \mathrm{Cu}_{1} \mathrm{~B}_{15}$ samples preheated up to 715 and $730 \mathrm{~K}$, measured at $475 \mathrm{~K}$. Crosses, experimental data; solid lines, fit using the experimental $F$; dashed lines, fit using the predicted $F$. Loops were measured for fields up to $2 \mathrm{kOe}$. For $730 \mathrm{~K}$, both fits overlap.

the experimental and predicted loops. This failure of the predicted $F$ in reproducing the experimental loop would be an indication that either the model is not accurate for that case, or as we will show for our studied samples, some modification has to be introduced to accurately reproduce the loop.

There can be several reasons which could justify that the model of superparamagnetic particles with dipolar interaction does not work for a specific sample, e.g., the strength of the interactions, which could force us to introduce higherorder terms in the Taylor expansions used in the model, or the presence of interactions of nondipolar origin, which was probably the case for the Mo-free alloy, since the coercivity maximum could not be reached due to the structural evolution of the samples. However, these reasons cannot be responsible for the behavior of the Mo-containing alloy preheated up to $715 \mathrm{~K}$, as in that case both the Curie temperature of the remaining amorphous matrix and the crystalline fraction are smaller than those of the sample preheated at $730 \mathrm{~K}$, and this would justify the opposite behavior (a stronger failure of the model for the $730 \mathrm{~K}$ sample, which is not the case).

Another possible effect that can be discarded is the presence of blocked particles in the studied samples. It has been shown ${ }^{23}$ that in the case of the coexistence of blocked and interacting superparamagnetic particles, the normalized $\Delta(m)$ curve $\left[\Delta_{N}(m)\right]$ for different measuring temperatures should not overlap, the blocked particles being responsible for remarkable deviations from the master curve behavior. This is certainly not the case, which is demonstrated in Fig. 7 where the overlapping is satisfied for the $730 \mathrm{~K}$ sample, while the $715 \mathrm{~K}$ sample shows only minor deviations from the master curve.

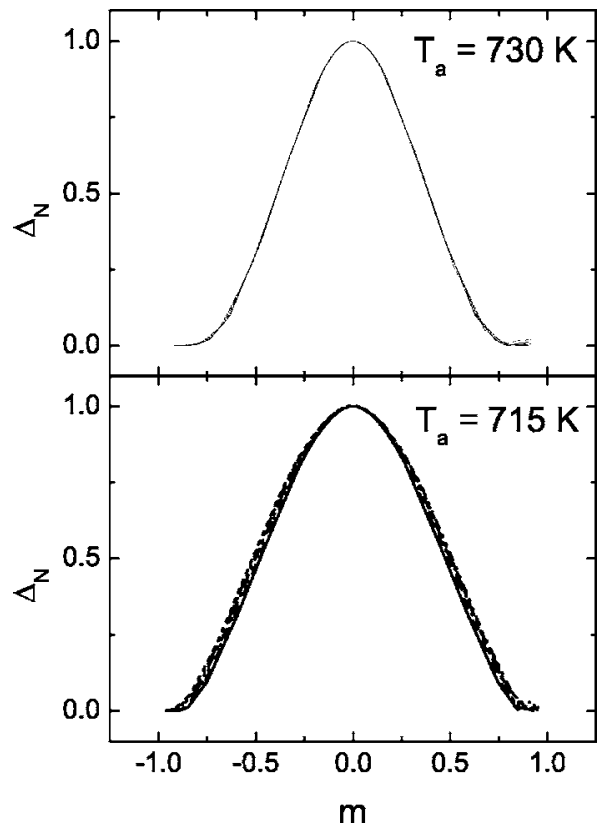

FIG. 7. Experimental $\Delta_{N}(m)$ of the $\mathrm{Fe}_{76} \mathrm{Cr}_{4} \mathrm{Mo}_{4} \mathrm{Cu}_{1} \mathrm{~B}_{15}$ samples preheated up to 715 and $730 \mathrm{~K}$, measured for several temperatures.

Therefore, we should consider the possibility that the behavior of the $715 \mathrm{~K}$ sample is due to a distribution of the magnetic moments in the sample, broader than in the case of the $730 \mathrm{~K}$ sample, which could be fitted with a single magnetic moment.

\section{B. Considering the grain size distribution}

According to the original model, ${ }^{5}$ the extension to a distribution of magnetic moments should be made by just using the mean magnetic moment in the expressions for the memory function. Therefore, the branches of the hysteresis loop can be obtained as

$$
M_{ \pm}=\sum_{i} N_{i} \mu_{i} m_{ \pm, i}
$$

where $N_{i}$ is the density of particles of each contribution, $\mu_{i}$ its magnetic moment, and

$$
m_{ \pm, i}=L\left(\frac{\mu_{i} H}{k T} \pm \frac{\mu_{i} H_{0}}{k T} F\left(m_{0}\right)\right)
$$

where $m_{0}$ indicates that the mean magnetic moment has been used in the expression for $F$. The interaction field for this system of particles would be defined as

$$
H_{0}=\frac{3 k T m_{r}}{\left\langle\mu_{a}\right\rangle},
$$

where $\left\langle\mu_{a}\right\rangle=\Sigma p_{i} \mu_{i}$ is the average magnetic moment of the particles, $p_{i}$ being the fraction of each contribution.

According to this reasoning, the half difference of the reduced magnetization curve should correspond to 


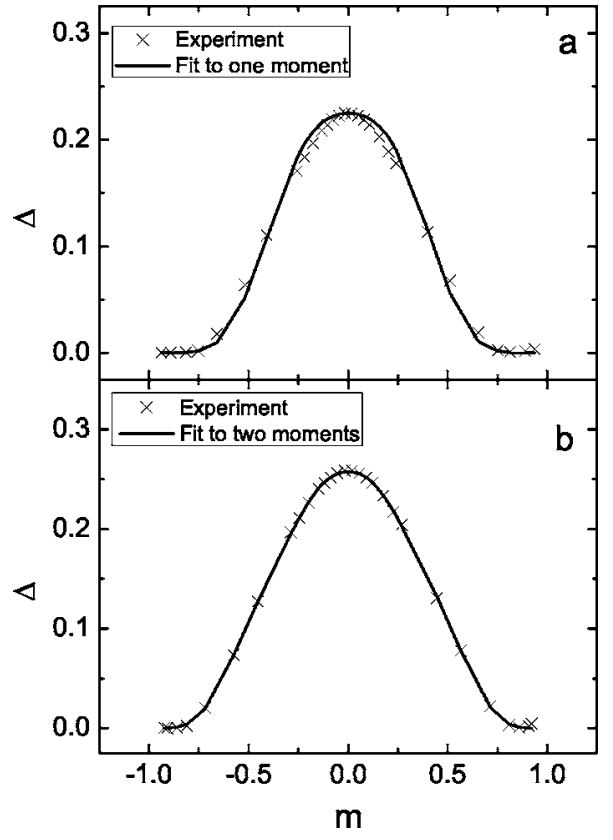

FIG. 8. Fit of $\Delta(m)$ of the $\mathrm{Fe}_{76} \mathrm{Cr}_{4} \mathrm{Mo}_{4} \mathrm{Cu}_{1} \mathrm{~B}_{15}$ sample preheated up to $715 \mathrm{~K}$ and measured at $475 \mathrm{~K}$, using one (a) or two (b) magnetic moments.

$$
\Delta=\frac{\left\langle\mu_{a}\right\rangle H_{0}}{k T} F\left(m_{0}\right) u^{\prime}\left(m_{0}\right)
$$

Therefore, according to the model, the $\Delta(m)$ curve corresponds to a single magnetic moment: the average value of the distribution. Figure 8(a) shows the fit to Eq. (10) of the experimental $\Delta(m)$ curve for the $715 \mathrm{~K}$ sample, evidencing that although there are some improvements with respect to Fig. 5, there are still important discrepancies between the experimental and predicted behavior for both high and low fields. The reason for the better fitting compared to Fig. 5 is that in the present case the least-squares fitting is applied directly to the $\Delta_{N}$ curve and therefore, the fitted curve goes above and below the experimental data in the different parts of the curve; in the case of Fig. 5, the fit was to the magnetization curve, making the fitted $\Delta(\mathrm{m})$ curve always below the corresponding experimental $\Delta(m)$ curve. It is also worth mentioning that the numerical procedure for fitting $\Delta_{N}$ uses $H$ as the abscissa axis. The reason for this is that $m$, the abscissa axis of these figures, depends on the previous determination of the magnetic moment of the particles, their number density, and the linear contribution previously mentioned. Therefore, minor differences in the determination of these parameters due to the different quality of the fittings produce the slight modifications in the shape of the $\Delta(\mathrm{m})$ curve, as evidenced in the figures.

To reduce the remaining discrepancies in the fitting of $\Delta$, let us consider that the origin of the hysteresis in this meanfield model is the interaction field $H_{0}$, which should depend on the average magnetic moment of the particles through Eq. (9) and is a measure of the dipolar field strength produced by the rest of the system (its physical meaning does not change with respect to the single-magnetic-moment model). The contribution of each component to the reduced magnetization loop can be rewritten from Eq. (6) by inserting into it the definition of $\Delta$ as the half difference between the branches of the loop:

$$
m_{ \pm, i}=L\left(\frac{\mu_{i} H}{k T}\right) \pm \Delta_{i}
$$

The hysteresis loop of the whole ensemble of particles can be expressed as

$$
m_{ \pm}=\sum_{i} p_{i} L\left(\frac{\mu_{i} H}{k T}\right) \pm p_{i} \Delta_{i}=m \pm \Delta .
$$

When considering that the way in which each particle responds to the mean interaction field (controlled by the cutoff function $F$ as well as by the mean interaction field) is a characteristic of the system and its mean magnetic moment, we arrive at Eq. (10) for the expression of $\Delta$, which has been shown not to be able to reproduce accurately the experimental data. However, as the cutoff function determining the response of the particle depends on the magnetic moment, we should consider that it is different for different particles. A natural conclusion of this reasoning is that the $\Delta(m)$ curve should be fitted by the sum of different contributions weighted according to the grain size distribution:

$$
\Delta=\sum_{i} p_{i} \Delta_{i}
$$

with

$$
\Delta_{i}=\frac{\mu_{i} H_{0}}{k T} F\left(m_{i}\right) u^{\prime}\left(m_{i}\right)
$$

Note that the magnetic moment of each particle is used for each contribution, in contrast to the original model, where the average magnetic moment was used.

To check these assumptions against the experimental data, we will represent the grain size distribution by only two components, in order to minimize number of the fitting parameters and yet allow a reasonable physical interpretation of the results. Figure 8(b) presents the fit of the experimental $\Delta(m)$ curve to Eq. (13) using two contributions. The magnetic moments of both components were extracted from the fit, and the consistency of the procedure has been checked by using those values to fit the anhysteretic magnetization curve (Fig. 9). It has to be noted that the linear contribution used in the fittings to represent the signal not coming from the nanoparticles does not influence the shape of the $\Delta(m)$ curve. Therefore, this fit involves one free parameter less than the Langevin fit to the anhysteretic curve. from

The branches of the hysteresis loop can be calculated

$$
M_{ \pm}=N_{1} \mu_{1} m_{ \pm, 1}+N_{2} \mu_{2} m_{ \pm, 2}
$$

with 

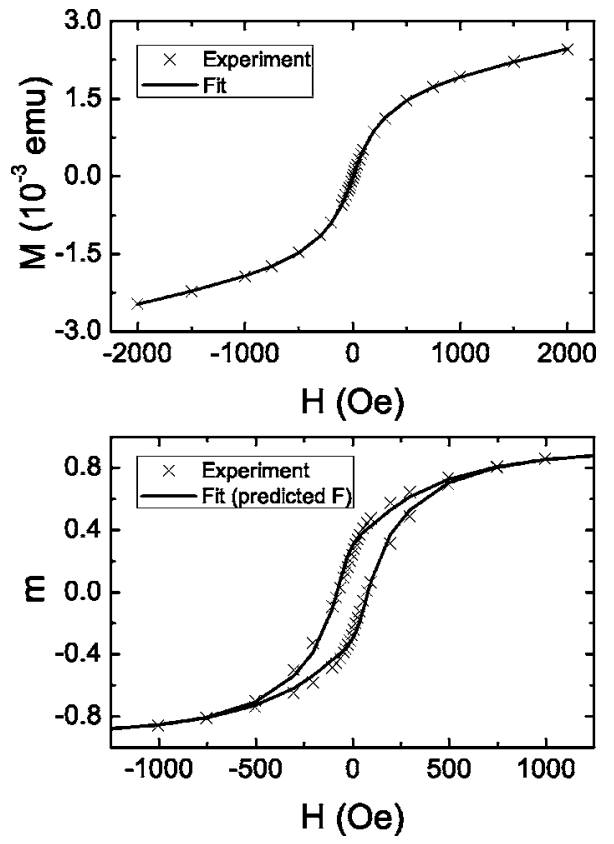

FIG. 9. Langevin fitting (upper) of the anhysteretic magnetization curve of the $\mathrm{Fe}_{76} \mathrm{Cr}_{4} \mathrm{Mo}_{4} \mathrm{Cu}_{1} \mathrm{~B}_{15}$ sample preheated up to $715 \mathrm{~K}$, using the same two magnetic moments obtained from the fitting of Fig. 8(b). Lower panel presents the corresponding reduced hysteresis loop. Crosses, experimental data; solid lines, fit.

$$
m_{ \pm, i}=L\left(\frac{\mu_{i} H}{k T} \pm \frac{\mu_{i} H_{0}}{k T} F\left(m_{i}\right)\right) .
$$

Figure 9 shows the experimental points together with the fit to the loop, evidencing the much better fit to the hysteresis loops by the modified model with the use of a grain size distribution (comparison should be made with the corresponding dashed loop of Fig. 6, as that is the one obtained from the predicted $F$ of a single average magnetic moment). The remaining differences between the fitted and experimental loops might originate from the simplified representation of the grain size distribution, with only two components. However, magnetic relaxation can also have an influence on these differences, ${ }^{24,25}$ although the loops have been measured at a reduced speed (about $150 \mathrm{~min}$ per hysteresis loop) and field increments from point to point are relatively small, to try to minimize that effect.

\section{TEMPERATURE DEPENDENCE OF THE MAGNETIC MOMENT OF THE NANOPARTICLES}

To be able to check how plausible these fitted magnetic moments are, we should first consider that the dipolar interactions provoke not only hysteresis but also a distortion of the temperature evolution of the magnetic moment of the particles. In fact, as the microstructure of the samples does not evolve in the considered temperature range, the observed increase of the magnetic moment as measuring temperature increases cannot be correlated with grain growth (Fig. 10). Therefore, we should consider that the moments obtained from the Langevin fittings are not the actual moments but the apparent ones.

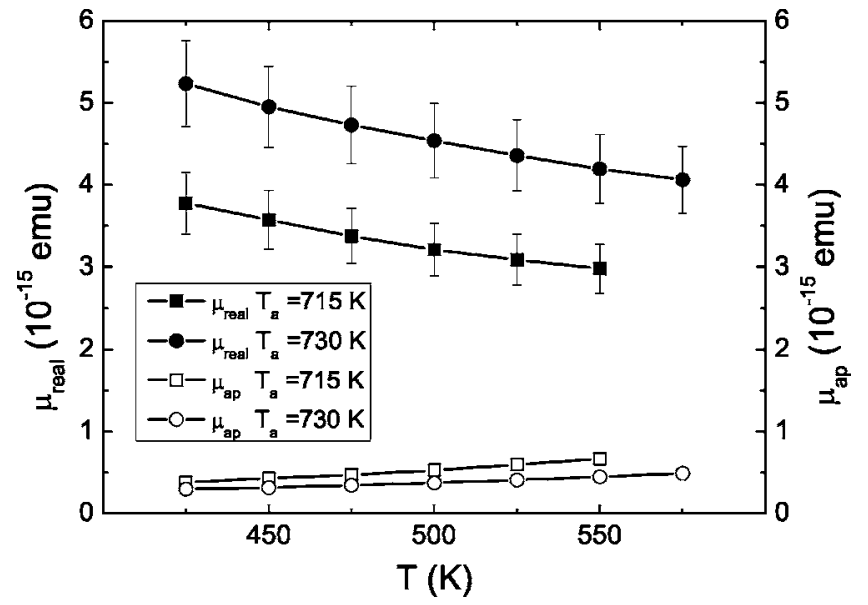

FIG. 10. Temperature dependence of the apparent (open, right axis) and real (solid, left axis) magnetic moments of the $\mathrm{Fe}_{76} \mathrm{Cr}_{4} \mathrm{Mo}_{4} \mathrm{Cu}_{1} \mathrm{~B}_{15}$ samples preheated up to 715 and $730 \mathrm{~K}$. Lines are a guide to the eyes. Error bars for the apparent magnetic moments are comparable to the size of the symbols.

It has been shown that this effect can be represented by an effective temperature $T^{*}$ which should be related to the dipolar interaction between the particles. ${ }^{8}$ Initially proposed as an independent model with respect to that of the hysteretic behavior of the nanoparticles mentioned above, there was a first attempt to correlate these physically interdependent behaviors, ${ }^{7}$ which produced plausible results. In the following, we will present a modification of this unified model, which solves some of the problems of the previous version.

Based on the fact that the calculated values of the saturation magnetization and the initial susceptibility of the sample should be independent of the representation that we make for the system (using the apparent $N$ and $\mu$ together with the actual temperature, or the real $N$ and $\mu$ with the help of an interaction temperature), the relationship between the apparent and real parameters is

$$
\begin{aligned}
& \mu_{a}=\frac{\mu}{1+\left(T^{*} / T\right)}, \\
& N_{a}=N\left(1+\frac{T^{*}}{T}\right) .
\end{aligned}
$$

The relationship between the two interaction models was based on the correlation between the thermal energy associated with the interaction temperature and the magnetic energy associated with the interaction field. Keeping this in mind, we should consider that this latter energy has to be related to the real magnetic moment of the particles in the presence of the interaction field, so we can set

$$
\xi \mu H_{0}=k T^{*} .
$$

Note that in the first attempt at correlating both models, the apparent moment was used. Although this difference is minute in the temperature region close to the pure superparamagnetic regime, as was the case in the $\mathrm{Cr}$-containing Finemet alloy studied in Ref. 7, the discrepancies get bigger 


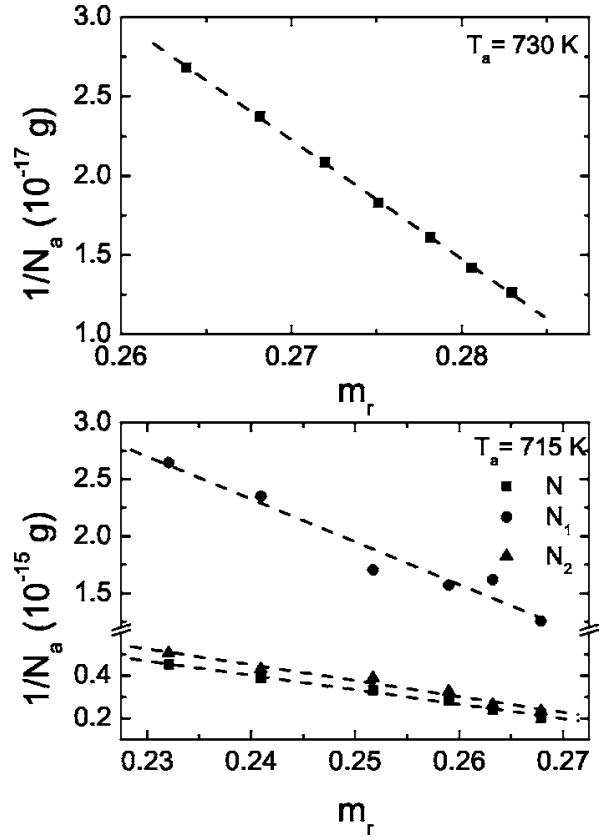

FIG. 11. Temperature dependence of the apparent density of particles of the $\mathrm{Fe}_{76} \mathrm{Cr}_{4} \mathrm{Mo}_{4} \mathrm{Cu}_{1} \mathrm{~B}_{15}$ samples preheated up to 715 and $730 \mathrm{~K}$. Lines are linear fits to the data. For the $715 \mathrm{~K}$ sample, the average density is plotted together with those of both contributions to the magnetic moment.

for a broader temperature range, as in this case (in the original paper, however, a deviation from the expected linear behavior was already noted in the region close to the coercivity maximum).

Taking into account that the moment of Eq. (5) is the apparent one (as it is the one extracted from the Langevin fit) and using Eq. (17), the interaction field can be expressed in terms of the interaction temperature and the real magnetic moment:

$$
H_{0}=\frac{3 k\left(T+T^{*}\right) m_{r}}{\mu} .
$$

Combining Eqs. (18)-(20), the relationship between the apparent and real density of particles is

$$
\frac{1}{N_{a}}=\frac{1}{N}\left(1-3 \xi m_{r}\right) .
$$

Therefore, the plot of the reciprocal apparent density of particles vs the reduced remanence of the loops should be a straight line from which the actual density of particles and the proportionality coefficient $\xi$ can be extracted (Fig. 11). In the case of the $715 \mathrm{~K}$ sample, the parameters corresponding to each of the magnetic contributions have been plotted, together with the average value of the density. The parameters extracted from the linear fits are shown in Table II. It is worth mentioning that all the linear fits produce the same value of the proportionality coefficient $\xi$ close to 1 . This suggests the accuracy of this approach, as the meaning of Eq. (19) is that both effects (hysteresis and tilt of the anhysteretic curve) are a representation of the dipolar interaction energy.
TABLE II. Real density of particles and proportionality factor in Eq. (21) for the $\mathrm{Fe}_{76} \mathrm{Cr}_{4} \mathrm{Mo}_{4} \mathrm{Cu}_{1} \mathrm{~B}_{15}$ samples preheated up to 715 and $730 \mathrm{~K}$. In the first case, the values corresponding to the average magnetic moment as well as to each contribution are presented.

\begin{tabular}{lll}
\hline \hline & $T_{a}=715 \mathrm{~K}$ & $T_{a}=730 \mathrm{~K}$ \\
\hline$N(1 / g)$ & $(5.0 \pm 0.2) \times 10^{14}$ & $(4.45 \pm 0.03) \times 10^{15}$ \\
$N_{1}(1 / g)$ & $(8.8 \pm 0.8) \times 10^{13}$ & \\
$N_{2}(1 / g)$ & $(4.5 \pm 0.3) \times 10^{14}$ & \\
$\xi$ & $1.11 \pm 0.09$ & $1.11 \pm 0.02$ \\
$\xi_{1}$ & $1.1 \pm 0.2$ & \\
$\xi_{2}$ & $1.1 \pm 0.2$ & \\
\hline
\end{tabular}

However, as the magnetic energy implies the average of the actual distribution of particles, it is reasonable that $\xi$ slightly departs from unity.

The real values of the magnetic moments (the average one in the case of the $715 \mathrm{~K}$ sample) are plotted in Fig. 10. As expected, the magnetic moment (grain size) increases with annealing temperature, while the magnetic moment decreases as measuring temperature increases. Assuming that the nanocrystals are of pure Fe, the average magnetic moments would correspond to mean grain sizes of 14 and 16 $\mathrm{nm}$, for the samples preheated at 715 and $730 \mathrm{~K}$, respectively. In the first case, the two contributions used for the fittings correspond to 13 and $17 \mathrm{~nm}$. These results are in agreement with a saturation of the grain size, which produces a narrowing of the grain size distribution for the higher annealing temperature. TEM observations (Fig. 12) show that in the sample preheated up to $715 \mathrm{~K}$ grains with significantly different sizes coexist, whereas for the sample preheated up to $730 \mathrm{~K}$ the grains are more uniform in size and the smaller ones are not detected. The maximum grain size found for the latter sample is of the order of $30 \mathrm{~nm}$. However, the grain

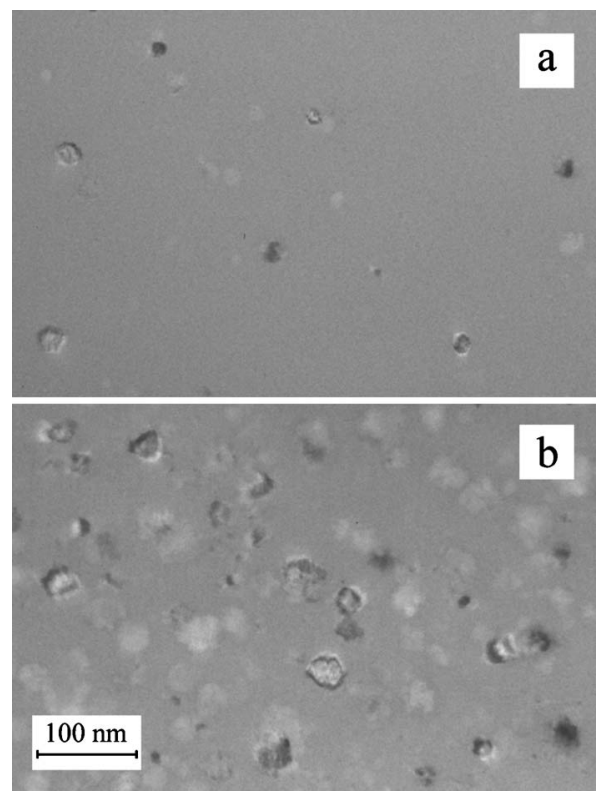

FIG. 12. TEM images of the samples preheated up to 715 (a) and 735 (b) K. 


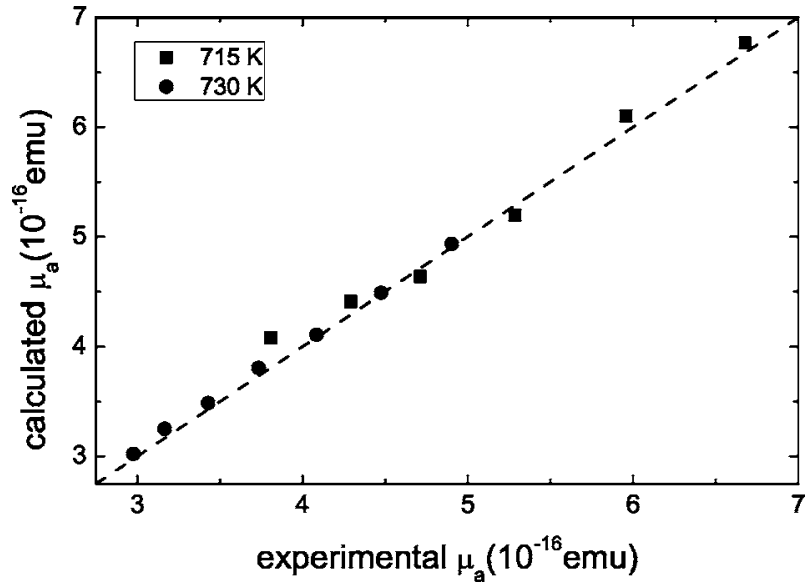

FIG. 13. Correlation between the apparent magnetic moment obtained from the Langevin fits and the apparent magnetic moment predicted by the model. Line corresponds to slope 1 .

sizes obtained by TEM are bigger than those calculated from the fittings of the magnetization curves, which is usual in this kind of study. ${ }^{8,18}$ It is worth mentioning that although the 30 $\mathrm{nm}$ grain size is considered as a limit diameter for singledomain Fe particles ${ }^{26}$ the actual value strongly depends on the geometry. ${ }^{27}$ Moreover, the relevant size for magnetic measurements is the magnetic size, which is below that limit. If particles were multidomain, the model would not be applicable, but also, coercivity should decrease with increasing grain size, which is the opposite behavior to the one presented in Fig. 3, supporting the applicability of the model.

As an internal check, once the interaction parameters are extracted from the fits and the real magnetic moments are calculated, Eq. (17) can be used to predict the apparent magnetic moments corresponding to these samples. Figure 13 shows the remarkable correlation between the apparent moments obtained from the Langevin fit of the experimental data and those predicted by the model.

\section{CONCLUSIONS}

The magnetic interactions between magnetic nanoparticles have been studied in Nanoperm-type alloys measured at high temperatures. As temperature is increased, these alloys show a transition from a soft magnetic behavior of par- ticles coupled by exchange to that of superparamagnetic particles with dipolar interaction, the transition being marked by a maximum in coercivity. The composition of the studied alloys was chosen in order to shift the coercivity maximum to lower temperatures, thus extending the temperature range where the interacting superparamagnetic particles can be studied.

The mean-field and effective temperature models of Allia et al. ${ }^{5,8}$ could be adapted to fit the observed behavior. It has been confirmed that at temperatures above the coercivity maximum, dipolar interactions between the superparamagnetic nanoparticles are responsible for both the hysteresis and the differences between the real magnetic moment of the nanoparticles and those apparent ones obtained from the Langevin fit of the magnetization curves.

While in the case of Cr-containing Finemet-type alloys the hysteresis loops could be fitted by using an average magnetic moment, in the case of Nanoperm-type alloys at early stages of nanocrystallization, a distribution of particle sizes has to be taken into account. It has been shown that the mean interaction field is related to the mean magnetic moment but the response of the system cannot be substituted by that of an average system: the response of the particles to the additional interaction field depends also on the magnetic characteristics of the particles.

The relationship between magnetic interaction energy and effective thermal energy has been refined to take into account the behavior of the system at temperatures more distant from the fully superparamagnetic regime. The consistency of the numerical procedure, shown by the correlation between the apparent magnetic moments obtained from the Langevin fits to the anhysteretic magnetization curves and the apparent magnetic moments predicted by the model, is increased with respect to the previous approaches to the problem.

\section{ACKNOWLEDGMENTS}

This work was supported by the Spanish Government and EU-FEDER (Project No. MAT 2004-04618), the PAI of Junta de Andalucía, the Hungarian Research Fund (OTKA Grants No. T-038383 and No. T-046795) and the HispanoHungarian Bilateral Cooperation Program (Grants No. HH2004-0015 and No. E-21/04). The authors are indebted to Dr. T. Kemény for fruitful discussion.
${ }^{1}$ P. Tartaj, M. P. Morales, T. González-Carreño, S. VeintemillasVerdaguer, and C. J. Serna, J. Magn. Magn. Mater. 290-291, 28 (2005).

${ }^{2}$ J. Dormann, D. Fiorani, and E. Tronc, Adv. Chem. Phys. 98, 283 (1997).

${ }^{3}$ J. Bansmann, S. H. Baker, C. Binns, J. A. Blackman, J.-P. Bucher, J. Dorantes-Dávila, V. Dupuis, L. Favre, D. Kechrakos, A. Kleibert, K.-H. Meiwes-Broer, G. M. Pastor, A. Perez, O. Toulemonde, K. N. Trohidou, J. Tuaillon, and Y. Xie, Surf. Sci. Rep. 56, 189 (2005).
${ }^{4}$ M. Vopsaroiu and P. R. Bissell, J. Phys. D 35, 1296 (2002).

${ }^{5}$ P. Allia, M. Coisson, M. Knobel, P. Tiberto, and F. Vinai, Phys. Rev. B 60, 12207 (1999).

${ }^{6}$ M. Knobel, W. C. Nunes, A. L. Brandl, J. M. Vargas, L. M. Socolovsky, and D. Zanchet, Physica B 354, 80 (2004).

${ }^{7}$ V. Franco, L. F. Kiss, T. Kemény, I. Vincze, C. F. Conde, and A. Conde, Phys. Rev. B 66, 224418 (2002).

${ }^{8}$ P. Allia, M. Coisson, P. Tiberto, F. Vinai, M. Knobel, M. A. Novak, and W. C. Nunes, Phys. Rev. B 64, 144420 (2001).

${ }^{9}$ M. Bahiana, J. P. Pereira Nunes, D. Altbir, P. Vargas, and M. 
Knobel, J. Magn. Magn. Mater. 281, 372 (2004).

${ }^{10}$ M. Miglierini, J. Degmová, T. Kanuch, and J. M. Greneche, Phys. Status Solidi A 201, 3280 (2004).

${ }^{11}$ T. Kemény, D. Kaptás, L. F. Kiss, J. Balogh, I. Vincze, S. Szabó, and D. L. Beke, Hyperfine Interact. 130, 181 (2000).

${ }^{12}$ M. E. McHenry and D. E. Laughlin, Acta Mater. 48, 223 (2000).

${ }^{13}$ M. Miglierini, M. Kopcewicz, B. Idzikowski, Z. E. Horváth, A. Grabias, I. Skorvánek, P. Duzewski, and Cs. S. Daróczi, J. Appl. Phys. 85, 1014 (1999).

${ }^{14}$ J. S. Blázquez, C. F. Conde, and A. Conde, Appl. Phys. Lett. 79, 2898 (2001).

${ }^{15}$ J. S. Blázquez, J. M. Borrego, C. F. Conde, A. Conde, and J. M. Greneche, J. Phys.: Condens. Matter 15, 3957 (2003).

${ }^{16}$ A. Hernando, M. Vázquez, T. Kulik, and C. Prados, Phys. Rev. B 51, 3581 (1995).

${ }^{17}$ A. Hernando, P. Marín, M. Vázquez, and G. Herzer, J. Magn. Magn. Mater. 177-181, 959 (1998).

${ }^{18}$ V. Franco, C. F. Conde, A. Conde, L. F. Kiss, D. Kaptás, T. Kemény, and I. Vincze, J. Appl. Phys. 90, 1558 (2001).
${ }^{19}$ A. Ślawska-Waniewska, M. Gutowski, H. Lachowicz, T. Kulik, and H. Matyja, Phys. Rev. B 46, 14594 (1992).

${ }^{20}$ L. del Riego, M. El Ghannami, M. Domínguez, C. F. Conde, and A. Conde, J. Magn. Magn. Mater. 196-197, 201 (1999).

${ }^{21}$ V. Franco, C. F. Conde, A. Conde, and L. F. Kiss, J. Magn. Magn. Mater. 215-216, 400 (2000).

${ }^{22}$ D. Kaptás, L. F. Kiss, J. Balogh, J. Gubicza, T. Kemény, and I. Vincze, Hyperfine Interact. 141/142, 175 (2002).

${ }^{23}$ P. Allia, M. Coisson, J. Moya, P. Tiberto, and F. Vinai, J. Magn. Magn. Mater. 254-255, 143 (2003).

${ }^{24}$ V. Basso, C. Beatrice, M. LoBue, P. Tiberto, and G. Bertotti, Phys. Rev. B 61, 1278 (2000).

${ }^{25}$ I. Skorvánek, J. Ková, and J. Jürgen Kötzler, J. Magn. Magn. Mater. 272-276, 1503 (2004).

${ }^{26}$ A. Herpin, Théorie du Magnétisme (Presses Universitaires de France, Paris, 1968), p. 752.

${ }^{27}$ R. C. O'Handley, Modern Magnetic Materials: Principles and Applications (Wiley, New York, 1999), p. 305. 